\title{
Biological Control of Botrytis cinerea by Volatiles of 'Isabella' Grapes
}

\author{
Eleni K. Kulakiotu, Constantine C. Thanassoulopoulos, and Evangelos M. Sfakiotakis
}

First and second authors: Laboratory of Plant Pathology; and third author: Laboratory of Pomology; Faculty of Agriculture, Aristotle University of Thessaloniki, Thessaloniki, 540 06, Greece.

Accepted for publication 23 March 2004.

\begin{abstract}
Kulakiotu, E. K., Thanassoulopoulos, C. C., and Sfakiotakis, E. M. 2004. Biological control of Botrytis cinerea by volatiles of 'Isabella' grapes. Phytopathology 94:924-931.

The effect of volatiles from cv. Isabella (Vitis labrusca) on the growth of Botrytis cinerea was tested in vitro and in situ, in the latter case on 'Roditis' grapes ( $V$. vinifera), at various temperatures. The goal of the research was to determine whether the volatiles emitted by Isabella grapes could be effective biocontrol agents of Botrytis cinerea. The closed

Mariotte system was used as a bioassay method to analyze quantitatively the biological action of these volatiles on fungal growth and disease development. The in vitro experiments revealed the inhibitory action of the Isabella volatiles on the sporulation and sclerotia formation of the fungus, as well as the stimulating action of the Roditis volatiles on the sporulation of the fungus. The in situ study confirmed the antifungal action of the Isabella volatiles as they reduced the inoculum and pathogenicity of $B$. cinerea. The antibiotic action was more pronounced at $21^{\circ} \mathrm{C}$. The study indicates that Isabella volatiles act as biocontrol agents of $B$. cinerea.
\end{abstract}

The amount of food lost annually worldwide during the postharvest period could feed between 200 and 300 million people for 1 year (29). Worldwide postharvest losses are estimated at 10 to $30 \%$ for most crops; in some perishable crops, losses as high as 30 to $50 \%$ are not unusual $(11,12,14,15,27,29,33,50)$. Conservative estimates put U.S. losses in perishable food crops due to postharvest diseases at $\approx 24 \%$ of the harvested crop (50). In spite of this, postharvest diseases have not received the attention that the magnitude of the problem warrants $(29,50)$. In all, $\approx 25$ species of fungi and bacteria are responsible for the major forms of decay in plant products after harvest (16). Among them, species of the genus Botrytis, and especially the species Botrytis cinerea Pers.:Fr., are major pathogens of stored and transported fruit, vegetables, ornamental crops, and nursery stocks (28). Botrytis rot was the most serious parasitic disease, detected in $32.5 \%$ of table grapes shipped to the New York market during the period 1972 to 1984 (8).

Control of Botrytis spp. in static and mobile storage conditions is achieved by a combination of chemical and physical methods (36). Reviews by Eckert and Sommer (17) and Eckert and Ogawa $(14,15)$ describe the development of control measures for most problems of stored fruit and vegetables. Fungicides are a primary means of controlling postharvest diseases, including diseases caused by Botrytis spp. The pronounced oncogenic risk of fungicides (37), the development of resistance in pathogens to fungicides applied for the control of postharvest diseases $(13,46)$, and the fact that, under optimal conditions for disease development, control by fungicides is often ineffective (48) decrease the effectiveness and desirability of chemical control of postharvest pathogens. Therefore, an urgent need exists for alternative and effective means of controlling postharvest diseases that pose less risk to human health and the environment (49-52). Baker (5) defines biological control of plant diseases as "the decrease of inoculum

Corresponding author: E. K. Kulakiotu; E-mail address: elenikk@ @agro.auth.gr

Publication no. P-2004-0623-01R

(c) 2004 The American Phytopathological Society or the disease-producing activity of a pathogen accomplished through one or more organisms, including the host plant but excluding man." According to Baker's broad definition of biological control, several promising approaches are available for developing new and potentially safer technologies for postharvest disease control (50), among which is the possibility of using natural plant volatiles.

Fries (21) defines volatile compounds as “...those compounds with a relatively high vapor pressure at physiological temperatures, which are capable of approaching an organism not only through the liquid phase but also through the gas phase. This is important, in particular to organisms like fungi which have virtually every single vegetative cell exposed to the surrounding medium and where, consequently, the surface to volume ratio is very high."

The present work involves the study of the effect of volatile substances emitted from 'Isabella' grapes as possible biocontrol agents of $B$. cinerea. The effect of Isabella volatiles on the growth of $B$. cinerea was tested in in vitro and in situ studies on 'Roditis' grapes at various temperatures. The closed Mariotte system was used as a bioassay method to analyze quantitatively the biological action of the volatiles on fungal growth. The study showed the decrease in inoculum and pathogenicity of $B$. cinerea through biological control by volatiles of the Isabella cultivar. Preliminary reports have been published (30-32).

\section{MATERIALS AND METHODS}

The fungus. $B$. cinerea was isolated from a naturally infected grape berry from the vineyards of the Aristotle University of Thessaloniki. This isolate of the fungus belongs to an intermediate sclerotial and sporulating morphotype (28). The fungus was grown on potato dextrose agar (PDA) medium (Oxoid Ltd., Hampshire, UK) in petri dishes (18 to $20 \mathrm{ml}$ of PDA per petri dish $90 \mathrm{~mm}$ in diameter). Cultures were obtained by transferring mycelium, $2 \mathrm{~mm}$ in diameter, from the margins of actively growing 4-day-old colonies. In order to minimize culture variability, inoculum was taken from the periphery of the colonies at the same distance from the center. 
Plant material. Clusters of cv. Isabella (Vitis labrusca L.) were collected from a vineyard in the area of Stavros, in the Pieria prefecture, and cv. Roditis ( $V$. vinifera L.) from a vineyard in the area of Orfani, in the Kavala prefecture, both located in the northern Greek province of Macedonia. Isabella is resistant to powdery mildew, downy mildew, and winter cold (22), as well as to $B$. cinerea, whereas Roditis is highly susceptible to $B$. cinerea. The clusters were selected arbitrarily from all parts of the vineyards. Clusters which were uniform in color and free of physiological irregularities and visible mycological, entomological, or other infections were selected. The ripening stage, expressed in soluble solids content, was 21.2 and $17.1 \%$ for Isabella and Roditis grape berries, respectively. The clusters were separated randomly into groups of smaller clusters.

Bioassay method. A modified closed Mariotte system (30), using 3-liter (first and third experiments) or 2-liter (second experiment) glass vessels, was used for all experiments. The vessels were placed in chambers at various temperatures $\left(21,10\right.$, and $0^{\circ} \mathrm{C}$ for 7, 15, and 75 days [first and third experiments] or for 25, 50, and 75 days [second experiment]), and then connected to the system. The closed Mariotte system allowed the concentration of $\mathrm{O}_{2}$ to be maintained at $20 \%$ and the removal of $\mathrm{CO}_{2}$, while it did not allow the exchange of gases (Fig. 1).

Effect on sporulation in vitro. Antifungal bioassay. Thirty-six 3-liter glass vessels were used as desiccators for the modified closed Mariotte system. A sterile conical flask containing $25 \mathrm{ml}$ of $4 \mathrm{~N}$ sodium hydroxide $(\mathrm{NaOH})$ was placed on the bottom of each of the 36 vessels to trap the respiratory $\mathrm{CO}_{2}$. Grape clusters also were placed on the bottom of 24 of the vessels. Five petri dishes inoculated with $B$. cinerea were placed over the fruit on sterile wire mesh screens. For the inoculation of the petri dishes, a B. cinerea mycelium, $5 \mathrm{~mm}$ in diameter, was transferred from the margins of actively growing 3-day-old colonies and placed upside down in the center of the dishes. Sterile filter papers $(5 \mathrm{~cm}$ by $5 \mathrm{~cm}$ [Whatman Chromatography paper $3 \mathrm{MM}$ ]) were placed in the base of the vessels and then moistened with $0.7 \mathrm{ml}$ of sterile water to provide high humidity. The treatments were as follows: (i) vessels with five inoculated petri dishes, (ii) vessels with five inoculated petri dishes and $200 \mathrm{~g}$ of Roditis grape berries in small clusters, and (iii) vessels with five inoculated petri dishes and $200 \mathrm{~g}$ of Isabella grape berries in small clusters. Four replicates were used for each treatment. Twelve vessels each were placed in one of the three temperatures described above. The 12 vessels then were connected to the closed Mariotte system. Colonies of the fungus were observed daily for possible differences in mycelial growth and morphology. For microscopic examination of the mycelial hyphae of the fungus, 108 slides in lactophenol blue were prepared with mycelial hyphae from the cultures of three of five plates from each of the 36 vessels.

To study the action of the grape volatiles on $B$. cinerea sporulation, the total number of conidia per plate was measured with a hematocytometer on the 7 th (at $21^{\circ} \mathrm{C}$ ), 15 th (at $10^{\circ} \mathrm{C}$ ), and 75 th (at $0^{\circ} \mathrm{C}$ ) day from the beginning of the experiment. Each plate was washed with $5 \mathrm{ml}$ of sterile water. The conidia suspension was filtered through cheesecloth to remove the hyphae. The action of the volatiles on sporulation was expressed as the average number of conidia per milliliter of water in four measurements per plate, for the 20 plates (five per replicate) per treatment, at each temperature.

To determine the nature of the action of the volatiles on the fungus's ability to produce conidia, PDA plates were inoculated with mycelial hyphae from the cultures of three plates from each vessel 7, 15, and 75 days from the beginning of the experiment. Plates were incubated at $21^{\circ} \mathrm{C}$. These new cultures from inocula which had been treated or nontreated with the volatiles were examined macroscopically daily to determine growth rate, sporulation, and sclerotia production.

Effect on the formation of sclerotia in vitro. Antifungal bioassay. For this experiment, 27 2-liter vessels were used: 9 ves- sels with $200 \mathrm{~g}$ of small clusters of Isabella, 9 vessels with $200 \mathrm{~g}$ of small clusters of Roditis, and 9 vessels without grapes of either cultivar. The treatments were the same as those of the previous experiment, with three replicates for each treatment. Three groups of nine vessels each were placed in chambers at 0,10 , and $21^{\circ} \mathrm{C}$. Each group of nine vessels was connected to the closed Mariotte system. Petri dishes were inoculated as described above and placed on wire mesh screens over grape clusters in each vessel.

The effect of the volatiles on the formation of $B$. cinerea sclerotia under the three temperatures was determined by examining each plate with a dissecting microscope $(\times 4) 25,50$, and 75 days from the beginning of the experiment. Criteria for evaluation of sclerotia production were the number, size, and color of the sclerotia. The sclerotia were ranked as large (diameter $\geq 3.5 \mathrm{~mm}$ ), medium (diameter $\geq 1.5 \mathrm{~mm}$ but $<3.5 \mathrm{~mm}$ ), and small (diameter $<1.5 \mathrm{~mm}$ ). Sclerotia also were characterized as black, gray, or white. The action of the volatiles on the formation of sclerotia was expressed as the arithmetical average of large and medium black sclerotia per plate, in a total of 15 plates (five per replicate) for each treatment at each of the three temperatures.

To evaluate the nature of the action of the volatiles on sclerotia formation, as in the case of fungus sporulation above, cultures of the fungus were obtained by inoculating PDA plates with $B$. cinerea mycelium which previously had been treated or nontreated with volatiles. $B$. cinerea mycelia were taken from cultures of four of five plates from each vessel 25, 50, and 75 days from the beginning of the experiment. Plates were incubated at $21^{\circ} \mathrm{C}$.

Effect on fungal growth in situ. Small grape clusters were randomly distributed in groups, with a total of 100 grape berries in each group. Grape berries were inoculated with mycelia to simulate natural infections of healthy plant organs in contact with infected dead organs $(24,28)$. The process of infection from a mycelium is essentially the same as that from germ tubes, although the mycelium almost always has a nutrient-providing saprophytic base (PDA disks here). Therefore, inoculum potential of mycelia is much greater than that of germinating conidia, and also is less dependent on the external environment (28). A $B$. cinerea mycelium, $5 \mathrm{~mm}$ in diameter, was transferred from the margins of actively growing 3 -day-old colonies and placed upside down on undamaged grape berries.

Antifungal bioassay. The same Mariotte system was used with treatments in three replicates as follows. Vessels with (i) clusters of 100 Isabella grape berries, 20 of which were inoculated with B. cinerea; (ii) clusters of 100 Roditis berries, 20 of which were inoculated with $B$. cinerea; and (iii) clusters of 100 Isabella berries, placed at the bottom of the vessels and clusters of 100 Roditis berries placed upon the Isabella clusters. Twenty berries of cv. Roditis also were inoculated with $B$. cinerea in treatment iii. The nine vessels were connected to the closed Mariotte system and the effect of the volatiles on the growth of the fungus in situ also was determined under the three temperatures. The experiment terminated on the 7 th day at $21^{\circ} \mathrm{C}$, the 15 th day at $10^{\circ} \mathrm{C}$, and the 75 th day at $0^{\circ} \mathrm{C}$. The number of (i) infected berries and (ii) in-

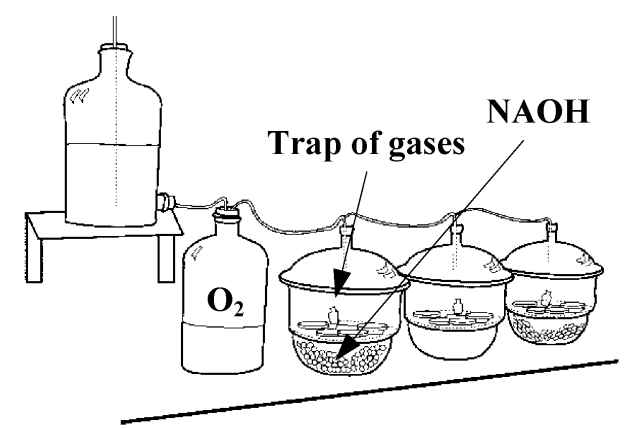

Fig. 1. Closed Mariotte system. 
fected berries on which there were fungal fruiting bodies was recorded. The latter observation was performed with a dissecting microscope $(x 4)$. The effect of the volatiles on the thallus of $B$. cinerea was determined by microscopic examination $(\times 40)$ of mycelial hyphae on slides mounted in lactophenol blue. The slides were made by transferring hyphae from seven berries from each vessel for a total of 189 observations.

Effect of volatiles on conidia production. The effect of the volatiles on the ability of $B$. cinerea to sporulate in situ on grapes was determined by estimating the number of conidia per berry, on seven berries from each vessel, by means of a hematocytometer, 7 (at $\left.21^{\circ} \mathrm{C}\right), 15\left(\right.$ at $\left.10^{\circ} \mathrm{C}\right)$, and $75\left(\right.$ at $0^{\circ} \mathrm{C}$ ) days from the beginning of the experiment. A total of 189 berries were used to measure the number of conidia produced by the fruiting bodies per berry. They were selected at random from the 20 berries that had been inoculated with the fungus. Each berry was washed with $10 \mathrm{ml}$ of sterile water thoroughly stirred in a beaker to separate the conidia from their conidiophores. The resulting conidial suspension was filtered through cheesecloth to remove hyphae. The density of the conidia was determined with a hematocytometer in four replicates.

Effect of volatiles on pathogenicity. In order to evaluate the action of the volatiles on the pathogenicity of $B$. cinerea under various temperatures, the amount of rotten tissue per berry was estimated in all 100 berries of each vessel. The severity of the disease or the extent of the plant tissue infected by $B$. cinerea was expressed as the percentage of the overall extent of plant tissue. The estimation of the percentage of rotten tissue was performed by notionally separating each berry into eight equal levels or sections along the vertical axis. When the decay had extended up to and including the second level of the berry, then the rotten tissue amounted to $25 \%$ of the total berry tissue; when the decay had extended up to and including the fourth level of the berry, then the rotten tissue amounted to $50 \%$ of the total berry tissue; and so on. The extent of the rot was expressed as the percentage of rotten tissue in the infected berries and of rotten tissue in the total num-

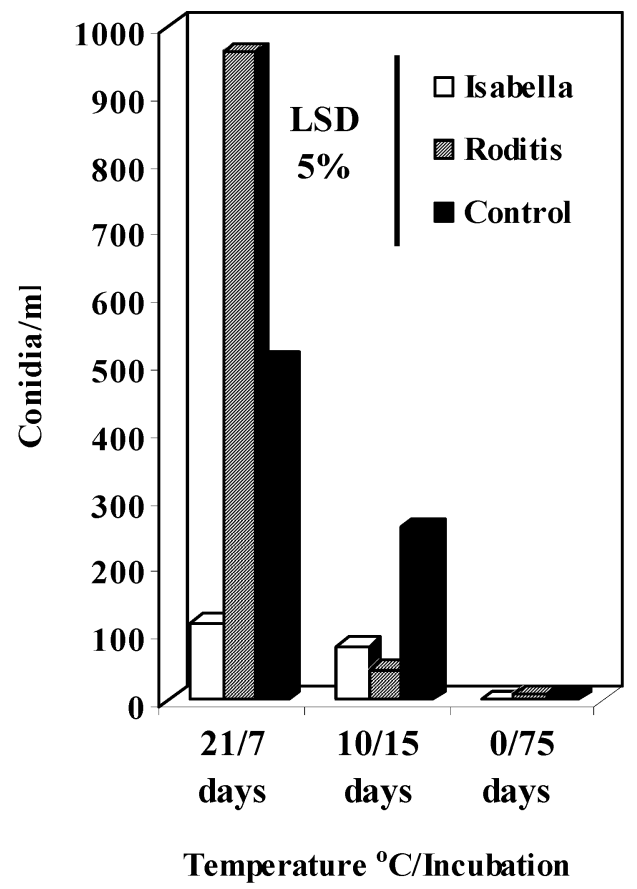

Fig. 2. Influence of the presence or absence of the volatile substances produced from grape berries of cvs. Isabella (Vitis labrusca) and Roditis (V. vinifera) on the production of conidia by Botrytis cinerea, at 21,10 , and $0^{\circ} \mathrm{C} \mathrm{7,15}$, and 75 days, respectively, after the inoculation of petri dishes and their insertion in the closed Mariotte system; least significant difference $\left(\mathrm{LSD}_{5 \%}\right)=240$. ber of berries per vessel, infected and noninfected (rotten tissue index). The latter expression of the extent of rot (rotten tissue index) equaled the product of multiplying the total number of levels or sections of the berries under decay by 100 , divided by the total number of levels of the berries (800).

Effect of volatiles on further growth of the fungus. In order to evaluate the residual effect of the volatiles on the growth of $B$. cinerea in vitro, mycelial hyphae of the fungus were transferred from berries in PDA plates 7 (at $21^{\circ} \mathrm{C}$ ), 15 (at $10^{\circ} \mathrm{C}$ ), and 75 (at $0^{\circ} \mathrm{C}$ ) days from the beginning of the experiment. The total number of PDA plates was 189 , representing the total number of randomly selected berries or sources of the inocula, per vessel ( 7 of 20 inoculated berries). These new cultures, from inocula that had been treated with the volatiles, were incubated at $21^{\circ} \mathrm{C}$ and examined macroscopically daily for 3 days to determine their growth rate $(\mathrm{mm} / \mathrm{h})$, and conidia and sclerotia production.

Data analysis. The analyses of data variance in the experiments were performed with the MSTAT (Michigan State University, version 4.00/EM) program. When the $F$ values were significant, the mean comparisons were performed with the least significant difference value, at the significance level of $P=0.05$.

\section{RESULTS}

Wherever they were present, Isabella grape berries showed strong antifungal action. The antifungal action was attributed to the presence of volatile substances emitted by these grapes.

Effect on sporulation in vitro. In order to prevent the volatiles from escaping, daily examination of the plates was performed without opening the vessels. The mycelial growth rate was the same in all treatments. Three days after the initiation of the experiment at $21^{\circ} \mathrm{C}, B$. cinerea covered the surface of the medium in all plates. Differences in the mycelial growth rate between the treatments were not observed at either 10 or $0^{\circ} \mathrm{C}$.

A dense, cottony mycelium grew in the plates of the vessels with clusters of Isabella, which indicated stimulation by the volatile substances. After 5 to 6 days, the same mycelium ceased to grow, followed by eventual collapse, with the formation of mycelial hyphae in layers upon the medium of the plate. The cultures assumed the characteristic gray color of $B$. cinerea, characteristic of the conidiophores that were forming. However, examination with a dissecting microscope showed that conidia had not formed at the edges of the conidiophores 7 days after the initiation of the experiment. Mycelia in the plates in the vessels with clusters of Roditis did not differ from that in the plates in the vessels with no clusters of either cultivar of grape in their interior (control). Sporulation of the fungus was observed after 7 days in the plates which had received the Roditis volatiles.

The microscopic examination of the hyphae showed endolysis of those from cultures which had been exposed to the volatiles from Isabella grapes. Secretion of the cell protoplast was observed without previous disruption of the cell walls. In addition to the lysis of the protoplast and the formation of large chemotopes, deformation of the cell walls also was observed. The above phenomena were more pronounced at $21^{\circ} \mathrm{C}$ than at either 10 or $0^{\circ} \mathrm{C}$.

After 7 days at $21^{\circ} \mathrm{C}$ in the presence of Isabella clusters in the vessels, the number of conidia of the $B$. cinerea cultures was significantly lower compared with the control (Fig. 2). In contrast, cultures of the fungus in the presence of Roditis clusters produced significantly more conidia compared with that of the control. After 15 days at $10^{\circ} \mathrm{C}$, the number of conidia produced by the fungus was not significantly affected by the volatiles of the Isabella and Roditis grapes compared with the control. After 75 days at $0^{\circ} \mathrm{C}$, the cultures of $B$. cinerea did not produce any conidia.

The growth rate of the new cultures of $B$. cinerea from inocula that had originated from cultures exposed to the volatiles of Isabella grapes, Roditis grapes, and the absence of grapes (control) 
was not affected. All the cultures were able to produce conidia and sclerotia at the same rate. All these cultures belonged to the same morphotype, with characteristics similar to those of the original culture.

Effect on formation of sclerotia in vitro. After 25 days at $21^{\circ} \mathrm{C}$, the presence of the Isabella and Roditis grapes significantly reduced the formation of sclerotia compared with the control. The presence of Isabella completely prevented the formation of sclerotia (Fig. 3). After 50 days at $10^{\circ} \mathrm{C}$, formation of sclerotia in all treatments was observed with no significant differences. However, after 75 days at $0^{\circ} \mathrm{C}$, significantly more sclerotia were produced in the absence of grapes than in the presence of Isabella or Roditis grapes (Fig. 3).

The new cultures of $B$. cinerea from inocula that had originated from cultures exposed to the volatiles of Isabella grapes, Roditis grapes, and the absence of grapes (control) all were able to produce sclerotia and conidia with characteristics similar to those of the morphotype of the original culture.

Effect on the growth of the fungus in situ. At all temperatures tested, the Isabella grape berries had almost no infection (Fig. 4). In contrast, the Roditis berries were highly infected with $90.0,66.3$, and $43.3 \%$ incidence of infection at 0,10 , and $21^{\circ} \mathrm{C}$, respectively. In the presence of Isabella volatiles, the incidence of infection in Roditis berries was significantly lower compared with the treatment without volatiles of Isabella grapes. The infection rates ranged from 43.0 to $16.3 \%$ at the above three temperatures.

No sporulation of the fungus was observed on the Isabella berries at any temperature (Fig. 5). In contrast, sporulation of $B$. cinerea was observed at $6.3,20.3$, and $22.6 \%$ at 0,10 , and $21^{\circ} \mathrm{C}$, respectively, on Roditis berries. In the presence of Isabella, sporulation on the Roditis berries was $4.3 \%$ at $21^{\circ} \mathrm{C}$ and $0 \%$ at the other two temperatures.

Microscopic examination of $B$. cinerea hyphae obtained from both Isabella and Roditis berries in the presence of Isabella volatiles showed endolysis. In contrast, hyphae obtained from the Roditis berries looked healthy and possessed conidiophores with abundant conidia. The lysis of the protoplast, the deformation of the cell walls, and the presence of large groups of chlamy-

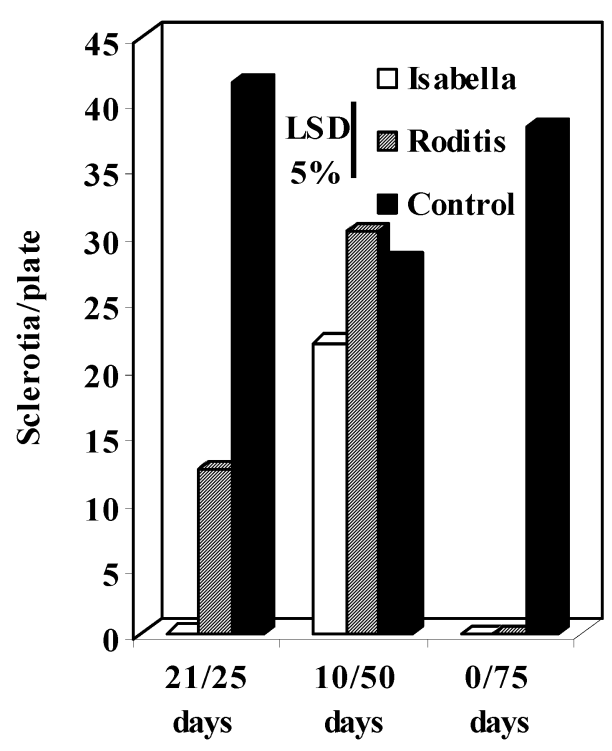

Temperature ${ }^{\circ} \mathrm{C} /$ Incubation

Fig. 3. Influence of the volatile substances of grape berries of cvs. Isabella (Vitis labrusca) and Roditis (V. vinifera) and of the absence of volatile substances (control) on the formation of sclerotia by Botrytis cinerea, at 21, 10 , and $0^{\circ} \mathrm{C}$ after 25,50 , and 75 , days, respectively; least significant difference $\left(\mathrm{LSD}_{5 \%}\right)=9.7$. dospores were the result of the action of the volatiles from Isabella berries on the mycelium of the fungus. These changes in the mycelial hyphae were more pronounced at $21^{\circ} \mathrm{C}$.

Effect on the amount of inoculum. Only at $21^{\circ} \mathrm{C}$ did the Roditis berries have a large number of conidia $(\approx 350 / \mathrm{ml})$. In all other cases (treatments and temperatures), the production of conidia on berries was very low, without significant differences (Fig. 6).

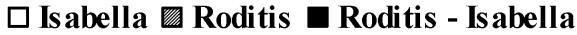

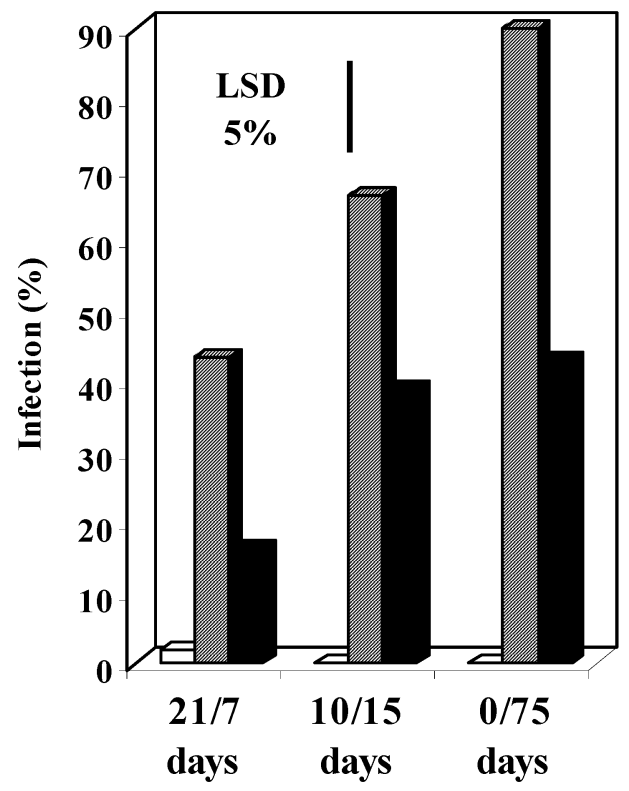

Temperature ${ }^{\circ} \mathrm{C} /$ Incubation

Fig. 4. Influence of temperature on infection of grape berries of cvs. Isabella (Vitis labrusca) and Roditis ( $V$. vinifera) and Roditis grapes in the presence of Isabella grapes by Botrytis cinerea 7, 15, and 75 days after the inoculation and their insertion in the closed Mariotte system; least significant difference $\left(\mathrm{LSD}_{5 \%}\right)=12.2$.

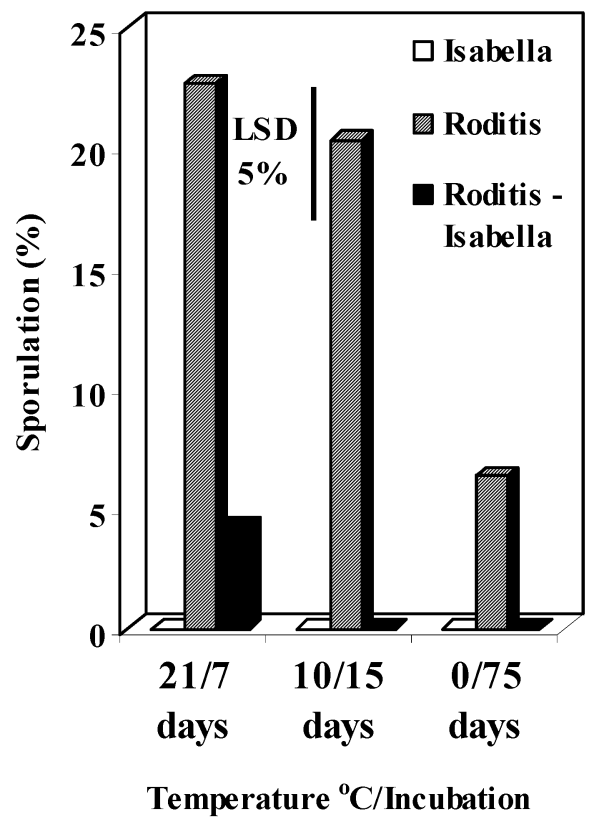

Fig. 5. Influence of temperature on sporulation of Botrytis cinerea on grape berries of cvs. Isabella (Vitis labrusca) and Roditis (V. vinifera) and on Roditis in the presence of Isabella grapes 7, 15, and 75 days after the inoculation and their insertion in the closed Mariotte system; least significant difference $\left(\mathrm{LSD}_{5 \%}\right)=4.76$. 
Effect on pathogenicity. There was no rotten tissue in the Isabella berries at any of the temperatures tested (Fig. 7). In contrast, the infected Roditis berries had 37.3, 62.0, and 59.6\% rotten tissue at 0,10 , and $21^{\circ} \mathrm{C}$, respectively. However, the Roditis berries in the presence of Isabella volatiles had significantly lower rates of rotten tissue at all temperatures.

The index of rotten tissue for the Isabella grapes was zero at all temperatures tested (Fig. 8). In contrast, the rotten tissue indices

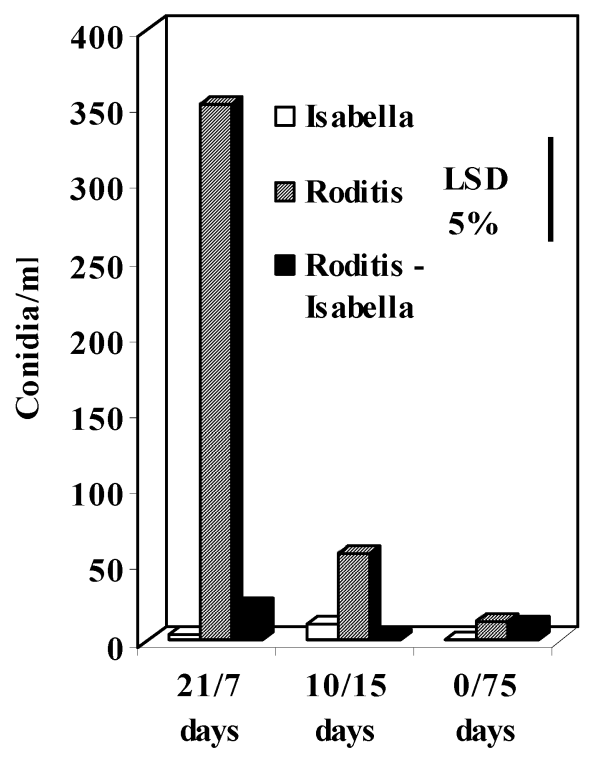

Temperature ${ }^{\circ} \mathrm{C} /$ Incubation

Fig. 6. Influence of temperature on the number of conidia of Botrytis cinerea on grape berries of cvs. Isabella (Vitis labrusca) and Roditis (V. vinifera) and on Roditis in the presence of Isabella grapes 7, 15, and 75 days after the inoculation and their insertion in the closed Mariotte system; least significant difference $\left(\operatorname{LSD}_{5 \%}\right)=61.9$.

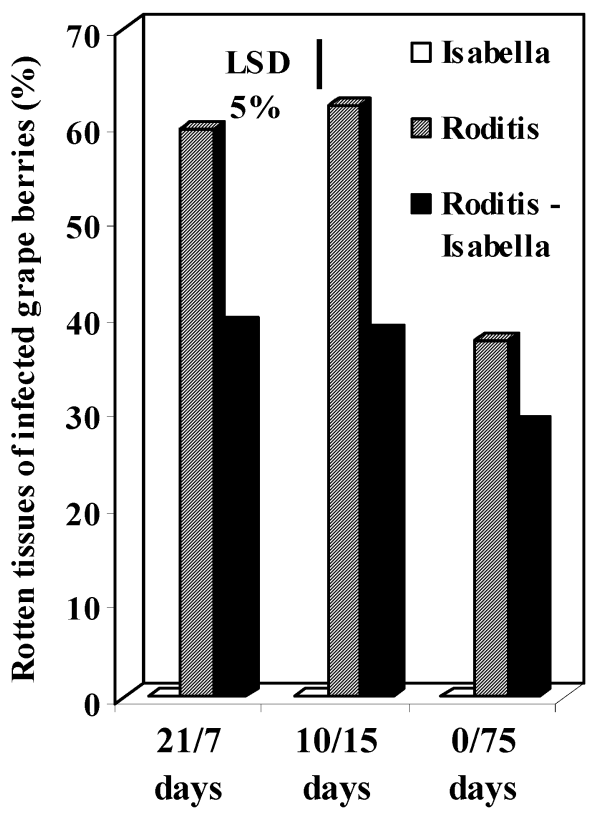

Temperature ${ }^{\circ} \mathrm{C} /$ Incubation

Fig. 7. Influence of temperature on the rotten tissue rate of the infected grape berries by Botrytis cinerea in grape berries of cvs. Isabella (Vitis labrusca) and Roditis (V. vinifera) and in Roditis in the presence of Isabella grapes 7, 15 , and 75 days after the inoculation and their insertion in the closed Mariotte system; least significant difference $\left(\mathrm{LSD}_{5 \%}\right)=4.34$. for the Roditis grape berries were 34,41 , and $36.3 \%$ at 0,10 , and $21^{\circ} \mathrm{C}$, respectively. However, in the presence of Isabella grapes, the Roditis grapes had significantly lower indices of rotten tissue (Fig. 8).

Effect of the action on the further growth of the fungus. At $10^{\circ} \mathrm{C}$, the growth rate $(\mathrm{mm} / \mathrm{h})$ of $B$. cinerea from hyphae from Isabella grapes was significantly lower $(P \leq 0.05[0.26])$ in relation to the other two treatments (i.e., cultures obtained from Roditis alone [0.48] or in the presence of the Isabella grapes [0.54]). There were no significant differences in the growth rate for cultures obtained from all the treatments at 21 and $0{ }^{\circ} \mathrm{C}$ (Fig. 9). The fungus always was able to sporulate and form sclerotia. All the cultures belonged to the morphotype of the original $B$. cinerea isolate.

\section{DISCUSSION}

The effect of the Isabella and Roditis volatiles on the growth of $B$. cinerea in in vitro and in situ studies, in the latter case on Roditis grapes, was tested at various temperatures. The volatiles from Isabella grapes were highly effective in suppressing $B$. cinerea under conditions optimal for the development of the pathogen. Although the temperature of $21^{\circ} \mathrm{C}$ is the optimum for the mycelial growth of $B$. cinerea (28), the fungus, in its effort to survive by creating abundant but ephemeral mycelial hyphae, eventually succumbed, because its plasmolyzed hyphae collapsed. At $21^{\circ} \mathrm{C}$, the endolysis $(6,10)$ of the hyphae which had been exposed to the Isabella volatiles was more pronounced. At this temperature, stimulation of mycelial growth was observed, which eventually led to the hyphae collapse in those cultures of the fungus that were growing under the influence of the Isabella volatiles. The above observation is similar to that of Harman et al. (26), who found that colonies of Alternaria alternata, in which sporulation is inhibited by plant-produced volatile substances, are characterized by a proliferation of aerial hyphae. The same researchers observed an unusual elongation of conidiophores, rising as aerial hyphae, and suggested that, because the concentrations

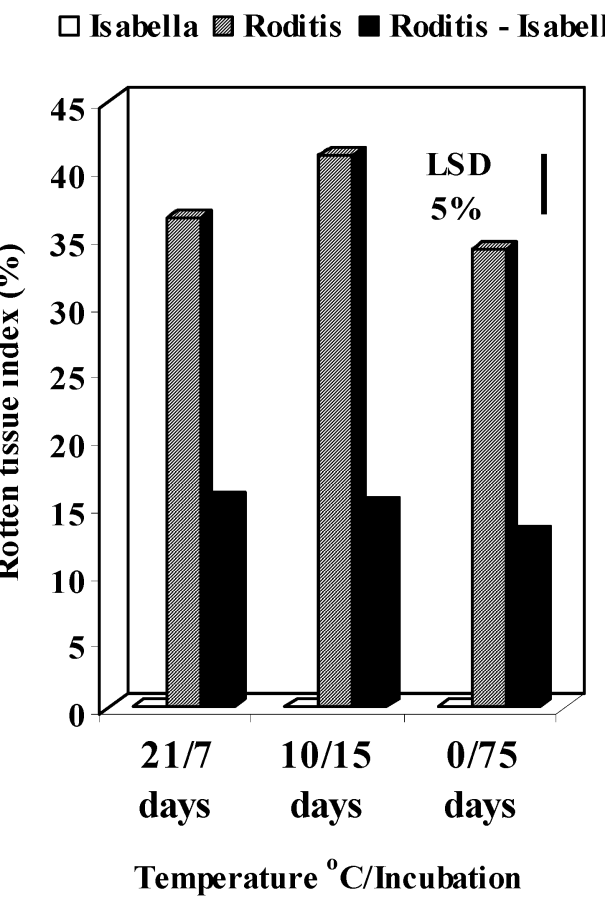

Fig. 8. Influence of temperature on the rotten-by Botrytis cinerea-tissue index of grape berries of cvs. Isabella (Vitis labrusca) and Roditis (V. vinifera) and of Roditis grapes in the presence of Isabella grapes 7, 15, and 75 days after the inoculation and their insertion in the closed Mariotte system; least significant difference $\left(\mathrm{LSD}_{5 \%}\right)=3.85$. 
of the volatiles were too low to have a pronounced nutritive effect, the volatiles act as fungal growth regulators. The activity of the volatile substances from the grapes, not only from the resistant Isabella but also from the susceptible Roditis, on conidia production by $B$. cinerea was the highest at $21^{\circ} \mathrm{C}$ (Fig. 2). The volatile substances from the resistant Isabella inhibited the sporulation of the fungus, while those of the susceptible Roditis stimulated it at $21^{\circ} \mathrm{C}$. Both effects indicate that the expression of Isabella resistance and Roditis susceptibility to $B$. cinerea was related to the inhibitory and stimulating actions, respectively, of the volatile substances of their grapes, without precluding the possibility that other factors were involved (38). They also indicate the important role of the volatile substances as inhibitors or stimulators of organisms $(19,21)$. At the same temperature, the Botrytis sp. was unable to form sclerotia and its hyphae collapsed through endolysis when the fungus was subjected to the antibiotic action of Isabella volatiles (Fig. 3). At $21^{\circ} \mathrm{C}, B$. cinerea treated with volatiles of the susceptible Roditis produced a high number of conidia and produced many fewer sclerotia compared with the control. This supports the theory that any factor which favors conidium production tends to reduce the production of sclerotia and vice versa (28).

Natural volatile compounds possessing in vitro antifungal activity may have potential as postharvest fumigants. The natural origin of the volatile compounds, their metabolism by fruit, their integral role in the human diet due to their presence in fresh fruit and vegetables, and their volatile nature may enhance consumer acceptance (3). Fallik et al. (18) concluded that the inhibitory effect of (E)-2-hexenal against $B$. cinerea at higher vapor-phase concentrations may be due, in part, to the severe damage to the fungal membranes and cell walls which resulted in collapse and deterioration of the hyphae. The results of the present study showed the strong in situ antibiotic action of the Isabella volatiles against $B$. cinerea, which, as in the in vitro study, is indicated by the endolysis of the mycelial hyphae $(7,23,34)$, the deformation of their cell walls, and the formation of chlamydospores. The antibiotic action of the Isabella volatiles led to the reduction of infection in the grape berries of the susceptible cv. Roditis and sporulation in the fungus on them, and the decrease in the number of conidia per grape berry and rot depth of the infected grape berries.

In the in situ experiment, the infection and sporulation rates of $B$. cinerea were significantly affected by the Isabella volatiles (Figs. 4 and 5). As in the in vitro experiments, the inhibitory action of the Isabella volatiles and the stimulating action of the Roditis volatiles were more pronounced at $21^{\circ} \mathrm{C}$. Generally, the effective suppression of plant diseases by biocontrol agents is largely affected by environmental conditions (24). Temperature affects the activity of the biocontrol agents. In this study, the antifungal action of the Isabella volatiles was less effective, but still significant, even at low temperatures, protecting the Roditis grapes from postharvest rotting by $B$. cinerea (Fig. 8).

The re-cultures of the fungus in PDA petri dishes grew normally and were morphologically identical to the original culture of the fungus, demonstrating the fungistatic action of the volatile substances from the Isabella grapes. However, according to Fries (21), whether volatile compounds are fungitoxic or fungistatic or even stimulating depends on their concentration and on the specific sensitivity of the responding fungus.

Volatiles of plants can have both an inhibitory and stimulating effect on the mycelial growth or sporulation and spore germination of pathogenic fungi (19-21), including B. cinerea-a pathogen of the grapevine (9) and the most serious rotting agent of grape berries (44). Natural plant volatiles also are implicated in plant resistance mechanisms $(2,48)$. The toxic activity of acetaldehyde on the growth of $B$. cinerea has been shown $(1,39,40)$, while the inhibitory action of volatiles like benzaldehyde and methyl salicylate on the growth of the same fungus also has been demonstrated (48). Aldehydes, including $\mathrm{C}_{6}$ and $\mathrm{C}_{9}$ compounds, inhibited the growth of the fungus (25). The inhibitory action of the vapors of aliphatic aldehydes, including acetaldehyde, propanal, butanal, and pentanal, against pathogenic fungi, including $B$. cinerea, also has been assessed (35). Benzaldehyde, 1-hexanol, E-2-hexenal, and 2-nonanone inhibited the growth of the fungus in vitro and the latter, selected as a starch-encapsulated, slowrelease antifungal compound, prevented the premature decay of stored fruits (47). The inhibitory action of the vapors of acetic acid on $B$. cinerea on tomato, grape, and kiwifruit also has been demonstrated (42). Song et al. (45) studied the inhibitory action of hexanal on the growth of the fungus in vitro and in vivo on apple slices. Archbold et al. (3) found that the natural volatile compounds hexanal, 1-hexanol, (E)-2-hexen-1-ol, (Z)-6-nonenal, (E)-3-nonen-2-one, methyl salicylate, and methyl benzoate possess potential activity as postharvest fumigants in the control of the fungus on strawberry, blackberry, and grape. Reddy et al. (41) studied the antifungal activity of essential oil volatiles of two clonal types of Thymus vulgaris against $B$. cinerea on strawberry. Fallik et al. (18) found that E-2 hexenal at a high concentration inhibited the growth of $B$. cinerea in vitro and in vivo on strawberry. Archbold et al. (4) found that the fumigation of bulk quantities of table grapes with the volatile (E)-2-hexenal can suppress mold effectively for up to 12 weeks of storage. We suggest that application of the volatile substances produced by cv. Isabella, following precise identification and appropriate synthesis, will give us an alternative and effective means of controlling postharvest diseases caused by $B$. cinerea. The coevolution and cohabitation of man with plants may have diminished the impact of toxic plant metabolites through the process of natural selection (51). Furthermore, it would seem that "natural" plant metabolites might be more biodegradable than synthetic ones (51).

With the exception of the sulfur dioxide treatment of table grapes after harvest (43), there are no commercially acceptable or safe postharvest treatments to control diseases of stored grapes (3). The potential health risk that the ingestion of sulfur dioxide poses for sulfite-sensitive individuals is recognized, and many uses of this chemical have been regulated (43). However, the

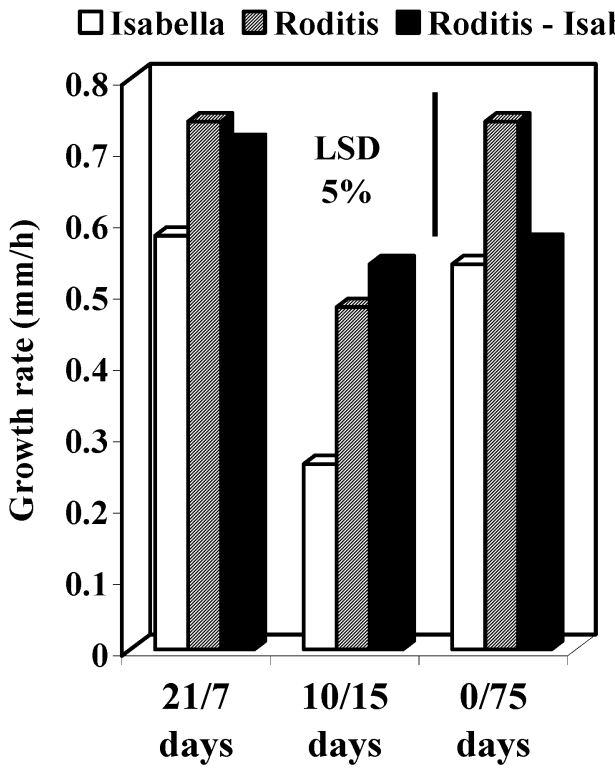

Temperature ${ }^{\circ} \mathrm{C} /$ Incubation

Fig. 9. Growth rate of re-cultures of Botrytis cinerea from inocula of hyphae taken from grape berries of cvs. Isabella and Roditis and Roditis in the presence of Isabella, which had been placed in the Mariotte system, at 21, 10, and $0^{\circ} \mathrm{C}$ for 7,15 and 75 days, respectively; least significant difference $\left(\operatorname{LSD}_{5 \%}\right)=0.18$. 
great advantage that $\mathrm{SO}_{2}$ has over other fungicides in relation to grapes is that it is effective as a volatile and the fruit does not have to be moistened. The results of our study suggest that the volatile substances of cv. Isabella are potentially useful as postharvest fumigants to control of $B$. cinerea on grapes.

\section{ACKNOWLEDGMENTS}

This research was made possible through the assistance of Euthymia Kulakiotu and Konstantinos Kulakiotis. We thank G. Holz for reviewing the manuscript; A. Siomos for his assistance with the statistical analyses; A. Mihalis, director of the University Studio Press, for his assistance in producing the drawing of the closed Mariotte system; and A. Hendry for proofreading the text.

\section{LITERATURE CITED}

1. Aharoni, Y., and Stadelbacher, G. J. 1973. The toxicity of acetaldehyde vapors to postharvest pathogens of fruits and vegetables. Phytopathology 63:544-545.

2. Andersen, R. A., Hamilton-Kemp, T. R., Hildebrand, D. F., McCracken, C. T., Jr., Collins, R. W., and Fleming, P. D. 1994. Structure-antifungal activity relationships among volatile $\mathrm{C}_{6}$ and $\mathrm{C}_{9}$ aliphatic aldehydes, ketones, and alcohols. J. Agric. Food Chem. 42:1563-1568.

3. Archbold, D. D., Hamilton-Kemp, T. R., Barth, M. M., and Langlois, B. E. 1997. Identifying natural volatile compounds that control gray mold (Botrytis cinerea) during postharvest storage of strawberry, blackberry, and grape. J. Agric. Food Chem. 45:4032-4037.

4. Archbold, D. D., Hamilton-Kemp, T. R., Clements, A. M., and Collins, R. W. 1999. Fumigating "Crimson Seedless" table grapes with (E)-2-hexenal reduces mold during long-term postharvest storage. (Abstr.) Hortscience 34:705-707.

5. Baker, K. F. 1987. Evolving concepts of biological control of plant pathogens. Annu. Rev. Phytopathol. 25:67-85.

6. Baker, K. F., and Cook, R. J. 1974. Biological Control of Plant Pathogens. Freeman \& Company, San Francisco.

7. Bruce, A., Austin, W. J., and King, B. 1984. Control of growth of Lentinus lepideus by volatiles from Trichoderma. Trans. Br. Mycol. Soc. 82:423-428.

8. Cappellini, R. A., Ceponis, M. J., and Lightner, G. W. 1986. Disorders in table grapes shipments to the New York market, 1972-1984. Plant Dis. 70:1075-1079.

9. Coertze, S., and Holz, G. 1999. Surface colonization, penetration, and lesion formation on grapes inoculated fresh or after cold storage with single airborne conidia of Botrytis cinerea. Plant Dis. 83:917-924.

10. Cook, R. J., and Baker, K. F. 1983. The Nature and Practice of Biological Control of Plant Pathogens. The American Phytopathological Society, St. Paul, MN.

11. Coursey, D. G. 1983. Post harvest losses in perishable foods of the developing world. Pages 485-514 in: Postharvest Physiology and Crop Preservation. M. Lieberman, ed. Plenum Press, New York.

12. Coursey, D. G., and Booth, R. H. 1972. The post-harvest phytopathology of perishable tropical produce. Rev. Plant Pathol. 51:751-765.

13. Delp, C. J. 1980. Coping with resistance to plant disease control agents. Plant Dis. 64:651-657.

14. Eckert, J. W., and Ogawa, J. M. 1985. The chemical control of postharvest diseases: Subtropical and tropical fruits. Annu. Rev. Phytopathol. 23:421-454.

15. Eckert, J. W., and Ogawa, J. M. 1988. The chemical control of postharvest diseases: Deciduous fruits, berries, vegetables and root/tuber crops. Annu. Rev. Phytopathol. 26:433-469.

16. Eckert, J. W., and Ratnayake, M. 1983. Host-pathogen interactions in postharvest diseases. Pages 247-264 in: Postharvest Physiology and Crop Preservation. M. Lieberman, ed. Plenum Press, New York.

17. Eckert, J. W., and Sommer, N. F. 1967. Control of diseases of fruits and vegetables by postharvest treatment. Annu. Rev. Phytopathol. 5:391-432.

18. Fallik, E., Archbold, D. D., Hamilton-Kemp, T. R., Clements, A. M., Collins, R. W., and Barth, M. M. 1998. (E)-2-hexenal can stimulate Botrytis cinerea growth in vitro and on strawberries in vivo during storage. J. Am. Soc. Hortic. Sci. 123:875-881.

19. French, R. C. 1985. The bioregulatory action of flavor compounds on fungal spores and other propagules. Annu. Rev. Phytopathol. 23:173199.

20. French, R. C., Long, R. K., Latterell, F. M., Graham, C. L., Smoot, J. J., and Shaw, P. E. 1978. Effect of nonanal, citral, and citrus oils on germination of conidia of Penicillium digitatum and Penicillium italicum. Phytopathology 68:877-882.
21. Fries, N. 1973. Effects of volatile organic compounds on the growth and development of fungi. Trans. Br. Mycol. Soc. 60:1-21.

22. Galet, P., and Morton, L. T. 1988. The family Vitaceae and Vitis speciation. In: Compendium of Grape Diseases. R. C. Pearson and A. C. Goheen, eds. The American Phytopathological Society, St. Paul, MN.

23. Gilbert, R. G., and Griebel, G. E. 1969. The influence of volatile substances from alfalfa on Verticillium dahliae in soil. Phytopathology 59:1400-1403.

24. Guetsky, R., Shtienberg, D., Elad, Y., and Dinoor, A. 2001. Combining biocontrol agents to reduce the variability of biological control. Phytopathology 91:621-627.

25. Hamilton-Kemp, T. R., McCracken, C. T., Jr., Loughrin, J. H., Andersen, R. A., and Hildebrand, D. F. 1992. Effects of some natural volatile compounds on the pathogenic fungi Alternaria alternata and Botrytis cinerea. J. Chem. Ecol. Vol. 18:1083-1091.

26. Harman, G. E., Mattick, L. R., Nash, G., and Nedrow, B. L. 1980. Stimulation of fungal spore germination and inhibition of sporulation in fungal vegetative thalli by fatty acids and their volatile peroxidation products. Can. J. Bot. 58:1541-1547.

27. Harvey, J. M. 1978. Reduction of losses in fresh market fruits and vegetables. Annu. Rev. Phytopathol. 16:321-41.

28. Jarvis, W. R. 1977. Botryotinia and Botrytis species: Taxonomy, physiology, and pathogenicity. Monograph No. 15. Research Branch Canada Department of Agriculture, Harrow, Ontario, Canada.

29. Kelman, A. 1989. Introduction: The importance of research on the control of postharvest diseases of perishable food crops. Phytopathology 79:1374

30. Kulakiotu, E. K. 2001. Natural volatile substances in the control of Botrytis cinerea. Ph.D. diss. Aristotle University of Thessaloniki, Greece.

31. Kulakiotu, E. K., Thanassoulopoulos, C. C., and Sfakiotakis, E. M. 2002. Influence of temperature in antimicrobial action of grape volatiles on in vitro growth of Botrytis cinerea. IOBC/WPRS Bull. Vol. 25:159162.

32. Kulakiotu, E. K., Thanassoulopoulos, C. C., and Sfakiotakis, E. M. 2002. Influence of temperature in antimicrobial action of grape volatiles on growth of Botrytis cinerea in vivo. IOBC/WPRS Bull. Vol. 25:163166.

33. Lieberman, M., ed. 1983. Postharvest Physiology and Crop Preservation. Plenum Press, New York.

34. Linderman, R. G., and Gilbert, R. G. 1969. Stimulation of Sclerotium rolfsii in soil by volatile components of alfalfa hay. Phytopathology 59:1366-1372.

35. Mattheis, J. P., and Roberts, R. G. 1993. Fumigation of sweet cherry (Prunus avium 'Bing') fruit with low molecular weight aldehydes for postharvest decay control. Plant Dis. 77:810-814.

36. Maude, R. B. 1980. Disease control. Pages 275-308 in: The Biology of Botrytis. J. R. Coley-Smith, K. Verhoeff, and W. R. Jarvis, eds. Academic Press, London.

37. National Research Council, Board on Agriculture. 1987. Regulating Pesticides in Food-The Delaney Paradox. National Academy Press, Washington, D.C.

38. Pezet, R., and Pont, V. 1992. Differing biochemical and histological studies of two grape cultivars in the view of their respective susceptibility and resistance to Botrytis cinerea. Pages 93-98 in: Proc. 10th Int. Botrytis Symp. Heraklion, Crete, Greece. Pudoc Scientific Publishers, Wageningen, The Netherlands.

39. Prasad, K., and Stadelbacher, G. J. 1973. Control of postharvest decay of fresh raspberries by acetaldehyde vapor. Plant Dis. Rep. 57:795-797.

40. Prasad, K., and Stadelbacher, G. J. 1974. Effect of acetaldehyde vapor on postharvest decay and market quality of fresh strawberries. Phytopathology 64:948-951.

41. Reddy, B. M. V., Angers, P., Gosselin, A., and Arul, J. 1998. Characterization and use of essential oil from Thymus vulgaris against Botrytis cinerea and Rhizopus stolonifer in strawberry fruits. Phytochemistry 47:1515-1510.

42. Sholberg, P. L., and Gaunce, A. P. 1995. Fumigation of fruit with acetic acid to prevent postharvest decay. Hortscience 30:1271-1275.

43. Smilanick, J. L., Hartsell, P. I., Henson, D., Fouse, D. C., Assemi, M., and Harris, C. M. 1990. Inhibitory activity of sulfur dioxide on the germination of spores of Botrytis cinerea. Phytopathology 80:217-220.

44. Snowdon, A. L. 1990. A Colour Atlas of Postharvest Diseases of Fruits and Vegetables. Volume 1: General Introduction \& Fruits. University of Cambridge Press, Wolfe Scientific, Barcelona, Spain.

45. Song, J., Leepipattanawit, R., Deng, W., and Beaudry, R. M. 1996. Hexanal vapor is a natural metabolizable fungicide: Inhibition of fungal activity and enhancement of aroma biosynthesis in apple slices. J. Am. Soc. Hortic. Sci. 121:937-942.

46. Spotts, R. A., and Cervantes, L. A. 1986. Populations, pathogenicity, and benomyl resistance of Botrytis spp., Penicillium spp., and Mucor piriformis in packinghouses. Plant Dis. 70:106-108. 
47. Vaughn, S. F., Spencer, G. F., and Shasha, B. S. 1993. Volatile compounds from raspberry and strawberry fruit inhibit postharvest decay fungi. J. Food Sci. 58:793-796.

48. Wilson, C. L., Franklin, J. D., and Otto, B. 1987. Fruit volatiles inhibitory to Monilinia fructicola and Botrytis cinerea. Plant Dis. 71:316319.

49. Wilson, C. L., and Pusey, P. L. 1985. Potential for biological control of postharvest plant diseases. Plant Dis. 69:375-378

50. Wilson, C. L., and Wisniewski, M. E. 1989. Biological control of posthar- vest diseases of fruits and vegetables: An emerging technology. Annu. Rev. Phytopathol. 27:425-441.

51. Wilson, C. L., Wisniewski, M. E., Biles, C. L., McLaughlin, R., Chalutz, E., and Droby, S. 1991. Biological control of postharvest diseases of fruits and vegetables: Alternatives to synthetic fungicides. Crop Prot. 10:172-177.

52. Wisniewski, M. E., and Wilson, C. L. 1992. Biological control of postharvest diseases of fruits and vegetables: Recent advances. Hortscience 27:94-98. 